\title{
Coupled fixed point theorems with applications to fractional evolution equations
}

\author{
He Yang ${ }^{1 *} \mathbb{D}$, Ravi P Agarwal ${ }^{2}$ and Hemant K Nashine ${ }^{2}$
}

\section{"Correspondence:} yanghe256@163.com

${ }^{1}$ College of Mathematics and Statistics, Northwest Normal University, Lanzhou, 730070, P.R. China

Full list of author information is available at the end of the article

\begin{abstract}
In this paper, we first prove some coupled fixed point theorems in partially ordered $\Phi$-orbitally complete normed linear spaces. And then apply the obtained fixed point theorems to a class of semilinear evolution systems of fractional order for proving the existence of coupled mild solutions under some weaker monotone conditions. An example is given to illustrate the application of the abstract results.
\end{abstract}

MSC: $34 \mathrm{~A} 08 ; 47 \mathrm{H} 10$

Keywords: coupled fixed point theorem; nonlinear fractional evolution system; equi-continuous $C_{0}$-semigroup; coupled mild solution; existence

\section{Introduction}

Let $E$ be a nonempty set, $\Psi: E \rightarrow E$ a mapping. If for $x \in E$, one has $\Psi x=x$, then $x \in E$ is called a fixed point of $\Psi$ in $E$. Fixed point theory plays an important role in nonlinear functional analysis. Different types of fixed point theorems have been used to prove the existence of solutions for differential and integral equations; see [1-17]. The Banach contraction principle is one of the most powerful fixed point theorems in nonlinear analysis for proving the existence and uniqueness of fixed points in metric spaces. It is interesting to improve and extend the conditions of the Banach contraction principle. Recently, by weakening the requirement on the contraction in partially ordered metric spaces, a series of fixed point theorems are established for monotone mappings by Agarwal et al. [1], Harjani and Sadarangani [7], Nieto and Rodriguez-López [12, 13], O'Regan and Petrusel [14] and Ran and Reurings [15].

We recall some definitions of the monotone mapping. A mapping $\Psi: E \rightarrow E$ is monotone means it is monotone nondecreasing or monotone nonincreasing. Assume that $(E, \leq)$ is a partially ordered set and $\Psi: E \rightarrow E$. For $x, y \in E$, if $x \leq y$ implies $\Psi(x) \leq \Psi(y), \Psi$ is called a monotone nondecreasing mapping in $E$. Similarly, we can define a monotone nonincreasing mapping in $E$. If for $x_{1}, x_{2} \in E, x_{1} \leq x_{2}$ implies $\Psi\left(x_{1}, y\right) \leq \Psi\left(x_{2}, y\right)$ for all $y \in E$, while for $y_{1}, y_{2} \in E, y_{1} \leq y_{2}$ implies $\Psi\left(x, y_{1}\right) \geq \Psi\left(x, y_{2}\right)$ for all $x \in E$, then we say that $\Psi$ is a mixed monotone mapping in $E$.

The above mentioned fixed point theorems, see $[1,7,12-15]$, are all for the monotone mapping. In [2], Bhaskar and Lakshmikantham extended the fixed point theorems obtained in $[1,7,12-15]$ to the mixed monotone mapping in partially ordered metric spaces. At first, they introduced a definition of the coupled fixed point. And then some coupled

(c) The Author(s) 2017. This article is distributed under the terms of the Creative Commons Attribution 4.0 International License (http://creativecommons.org/licenses/by/4.0/), which permits unrestricted use, distribution, and reproduction in any medium, provided you give appropriate credit to the original author(s) and the source, provide a link to the Creative Commons license, and indicate if changes were made. 
fixed point theorems were proved in partially ordered metric spaces. Recently, these coupled fixed point theorems were refined and improved by Lakshmikantham and Ćirić [9], Luong and Thuan [11] and Samet [16].

In the above mentioned results, the assumptions of mixed monotone property and contraction property of the mapping $\Psi$ are essential. The purpose of this paper is to delete or weaken these conditions. In this paper, by utilizing a different technique, we prove some coupled fixed point theorems in a partially ordered $\Phi$-orbitally complete normed linear space. In our results, we neither assume that the mapping $\Psi$ is mixed monotone, nor assume that it is a contraction. We divide the mapping $\Psi$ into two parts, and assume that every part satisfies some conditions, by using an existing Krasnoselskii-type fixed point theorem, a coupled fixed point theorem for the mapping $\Psi$ is proved. As applications, we apply the obtained coupled fixed point theorem to a certain abstract fractional evolution systems for proving the existence of coupled mild solutions.

The rest of this paper is organized as follows. In Section 2, some definitions are recalled and an existing Krasnoselskii-type fixed point theorem is introduced. In Section 3, coupled fixed point theorems are proved. In Section 4, we apply the obtained coupled fixed point theorem to a certain abstract fractional evolution systems. A specific example is given in Section 5 to illustrate the abstract results.

\section{Preliminaries}

Let $E$ be a partially ordered normed linear space with partial order $\leq$ and the norm $\|\cdot\|_{E}$. If two elements $x, y \in E$ satisfy either $x \leq y$ or $x \geq y$, we say that they are comparable. If $E$ is complete with respect to the norm $\|\cdot\|_{E}$, we called it a partially ordered complete normed linear space.

Definitions 2.1-2.5 can be found in $[4,5]$.

Definition 2.1 Let $\Psi: E \rightarrow E$ be a mapping. For any $x \in E$, we define an orbit $\Phi(x ; \Psi)$ by

$$
\Phi(x ; \Psi)=\left\{x, \Psi x, \Psi^{2} x, \ldots, \Psi^{n} x, \ldots\right\} .
$$

If for any sequence $\left\{x_{n}\right\} \subset \Phi(x ; \Psi), x_{n} \rightarrow x^{*}$ implies $\Psi x_{n} \rightarrow \Psi x^{*}$ for each $x \in E, \Psi$ is said to be $\Phi$-orbitally continuous in $E$. A normed linear space $\left(E,\|\cdot\|_{E}\right)$ is called $\Phi$-orbitally complete if every Cauchy sequence $\left\{x_{n}\right\} \subset \Phi(x ; \Psi)$ converges to a point $x^{*}$ in $E$.

Definition 2.2 A function $\phi: \mathbb{R}^{+} \rightarrow \mathbb{R}^{+}$is called a $\mathfrak{D}$-function if it is a monotone nondecreasing and upper semi-continuous function satisfying $\phi(0)=0$.

Definition 2.3 A mapping $\Psi: E \rightarrow E$ is called partially nonlinear $\mathfrak{D}$-Lipschitz if for all comparable elements $x, y \in E$, there is a $\mathfrak{D}$-function $\phi: \mathbb{R}^{+} \rightarrow \mathbb{R}^{+}$such that

$$
\|\Psi x-\Psi y\|_{E} \leq \phi\left(\|x-y\|_{E}\right)
$$

Furthermore, if $\phi(r)<r$ for $r>0, \Psi$ is called a partially nonlinear $\mathfrak{D}$-contraction in $E$.

Definition 2.4 A mapping $\Psi: E \rightarrow E$ is said to be partially compact if for all totally ordered sets or chains $C \subset E, \Psi(C)$ is a relatively compact subset of $E$. 
Definition 2.5 The norm $\|\cdot\|_{E}$ and the order relation $\leq$ on a partially ordered normed linear space $\left(E, \leq,\|\cdot\|_{E}\right)$ are said to be compatible if for any monotone sequence $\left\{x_{n}\right\}$ in $E$, subsequence $\left\{x_{n_{k}}\right\}$ of $\left\{x_{n}\right\}$ converges to $x^{*}$ implies that the whole sequence $\left\{x_{n}\right\}$ converges to $x^{*}$.

Remark 2.6 From Definition 2.1, if the normed linear space $\left(E,\|\cdot\|_{E}\right)$ is complete, then it is $\Phi$-orbitally complete. But the converse expression may not be true.

Remark 2.7 The $\mathfrak{D}$-functions and the partially nonlinear $\mathfrak{D}$-Lipschitz conditions are much useful in research of solutions for nonlinear differential equations via fixed point theorems; see [6].

The following Krasnoselskii-type fixed point theorem was proved by Dhage in [4].

Lemma 2.8 Let $E$ be a partially ordered complete normed linear space with the partial order $\leq$ and the norm $\|\cdot\|_{E}$ such that $\leq$ and $\|\cdot\|_{E}$ are compatible. Suppose that $A_{1}, A_{2}$ : $E \rightarrow E$ are two monotone nondecreasing mappings satisfying:

(a) $A_{1}$ is continuous and a partially nonlinear $\mathfrak{D}$-contraction,

(b) $A_{2}$ is continuous and partially compact,

(c) there is an element $v_{0} \in E$ satisfying $v_{0} \leq A_{1} v_{0}+A_{2} y$ for all $y \in E$, and

(d) every pair of elements has an upper and a lower bound in $E$.

Then $x=A_{1} x+A_{2} x$ has a solution in $E$.

\section{Fixed point theorems}

Let $\left(E,\|\cdot\|_{E}\right)$ be a $\Phi$-orbitally complete normed linear space. Define a positive cone $K$ in Eby

$$
K=\{x \in E: x \geq 0\} .
$$

Then $E$ becomes now a partially ordered $\Phi$-orbitally complete normed linear space with the partial order $\leq$ induced by $K$. It is well known that the partially order $\leq$ and the norm $\|\cdot\|_{E}$ are compatible if cone $K$ is normal.

By Lemma 2.8, we first prove the following fixed point theorem.

Theorem 3.1 Let E be a partially ordered $\Phi$-orbitally complete normed linear space with the norm $\|\cdot\|_{E}$ and the partial order $\leq$, whose positive cone $K$ is normal, and let $D$ be a nonempty closed subset of $E$. Assume that $A_{1}, A_{2}: D \rightarrow D$ are two monotone nondecreasing mappings satisfying:

(a) $A_{1}$ is $\Phi$-orbitally continuous and

$$
\left\|A_{1} x-A_{1} y\right\|_{E}<\|x-y\|_{E}
$$

for all comparable elements $x, y \in D$ with $x \neq \equiv y$,

(b)' $A_{2}$ is $\Phi$-orbitally continuous and partially compact, 
(c) there exists an element $v_{0} \in D$ such that $v_{0} \leq A_{1} v_{0}+A_{2} y$ for all $y \in D$, and

(d) every pair of elements in $D$ has an upper and a lower bound.

Then the equation $x=A_{1} x+A_{2} x$ has a solution in $D$.

Proof By Remark 2.7 of [4] and the proof of Lemma 2.8, if the continuity of operators $A_{1}$ and $A_{2}$ is replaced by the $\Phi$-orbitally continuity in conditions (a) and (b) of Lemma 2.8, the conclusion of Lemma 2.8 is still true. On the other hand, since $D$ is a nonempty closed subset of $E$, it follows that $D$ is $\Phi$-orbitally complete and has partial order $\leq$. For any comparable elements $x, y \in D, x \neq \equiv$, by the condition (a)', it follows that there is $\tau \in(0,1)$ satisfying

$$
\left\|A_{1} x-A_{1} y\right\|_{E} \leq \tau\|x-y\|_{E}<\|x-y\|_{E}
$$

Let $\phi(r)=\tau r$. Then $\phi$ is a $\mathfrak{D}$-function and $\phi(r)<r$ for any $r>0$. This implies that the condition (a) of Lemma 2.8 is satisfied. By Lemma 2.8, we obtain the desired conclusion.

If the inequality given in assumption (c) of Lemma 2.8 is reverse, more precisely, the condition (c) of Lemma 2.8 is replaced by

(c)' there is $v_{0} \in D$ satisfying $v_{0} \geq A_{1} v_{0}+A_{2} y$ for all $y \in D$.

By Theorem 3.7 of [4], the conclusion of Lemma 2.8 is still true. Hence we can obtain the following fixed point theorem. Since the proof is similar to Theorem 3.1, we omit the details here.

Theorem 3.2 Let E be a partially ordered $\Phi$-orbitally complete normed linear space with the norm $\|\cdot\|_{E}$ and the partial order $\leq$, whose positive cone $K$ is normal, and let $D$ be a nonempty closed subset of $E$. Assume that $A_{1}, A_{2}: D \rightarrow D$ are two monotone nondecreasing mappings satisfying the conditions $(a)^{\prime},(b)^{\prime},(c)^{\prime}$ and $(d)$. Then $x=A_{1} x+A_{2} x$ has a solution in $D$.

Let $\widehat{E}:=E \times E$. Define a sum and a scalar multiplication in $\widehat{E}$ by

$$
\begin{aligned}
& w+v=\left(w_{1}, w_{2}\right)+\left(v_{1}, v_{2}\right)=\left(w_{1}+v_{1}, w_{2}+v_{2}\right), \\
& \lambda w=\lambda\left(w_{1}, w_{2}\right)=\left(\lambda w_{1}, \lambda w_{2}\right)
\end{aligned}
$$

for all $w=\left(w_{1}, w_{2}\right), v=\left(v_{1}, v_{2}\right) \in \widehat{E}$ and $\lambda \in \mathbb{R}$. And define a positive cone and a norm in $\widehat{E}$ by

$$
\begin{aligned}
& K_{\widehat{E}}=\left\{w=\left(w_{1}, w_{2}\right) \in \widehat{E}: w_{1}, w_{2} \in K\right\}, \\
& \|v\|_{\widehat{E}}=\left\|\left(v_{1}, v_{2}\right)\right\|_{\widehat{E}}=\left\|v_{1}\right\|_{E}+\left\|v_{2}\right\|_{E}, \quad v=\left(v_{1}, v_{2}\right) \in \widehat{E} .
\end{aligned}
$$

Then $\left(\widehat{E},\|\cdot\|_{\widehat{E}}\right)$ is a partially ordered normed linear space with the order relation $\leq$ induced by $K_{\widehat{E}}$. Let $D$ be a nonempty closed subset of $E$. Then $D \times D$ is a nonempty closed subset of $\widehat{E}$. It is clear that cone $K_{\widehat{E}}$ is normal if $K$ is normal. 
Let $Q: \widehat{E} \rightarrow E$. A pair of elements $(x, y) \in \widehat{E}$ is called a coupled fixed point of $Q: \widehat{E} \rightarrow E$ if and only if it satisfies

$$
x=Q(x, y), \quad y=Q(y, x) .
$$

By Theorem 3.1, the following coupled fixed point theorem is obtained.

Theorem 3.3 Let E be a partially ordered $\Phi$-orbitally complete normed linear space with the partial order $\leq$ and the norm $\|\cdot\|_{E}$, whose positive cone $K$ is normal, and let $D$ be a nonempty closed subset of $E$. Assume that $A_{1}, A_{2}: D \rightarrow D$ are two monotone nondecreasing mappings satisfying

(i) $A_{1}$ is $\Phi$-orbitally continuous and a partially nonlinear $\mathfrak{D}$-contraction,

(ii) $A_{2}$ is $\Phi$-orbitally continuous and partially compact,

(iii) there is an element $v_{0} \in D$ satisfying $v_{0} \leq A_{1} v_{0}+A_{2} y$ for all $y \in D$, and

(iv) every pair of elements in $D$ has an upper and a lower bound.

Then $Q(x, y)=A_{1} x+A_{2} y$ has a coupled fixed point in $\widehat{E}$.

Proof Since $\left(E, \leq,\|\cdot\|_{E}\right)$ is a partially ordered $\Phi$-orbitally complete normed linear space, and positive cone $K$ is normal, it follows that $\left(\widehat{E}, \leq,\|\cdot\|_{\widehat{E}}\right)$ is a partially ordered $\Phi$-orbitally complete normed linear space and positive cone $K_{\widehat{E}}$ is normal. Since $D$ is a nonempty closed subset of $E$, it follows that $D \times D$ is a nonempty closed subset of $\widehat{E}$.

Let $\widehat{D}=D \times D$. Define two operators $\widehat{A}_{1}, \widehat{A}_{2}: \widehat{D} \rightarrow \widehat{D}$ by

$$
\widehat{A}_{1} u=\left(A_{1} x, A_{1} y\right), \quad \widehat{A}_{2} u=\left(A_{2} y, A_{2} x\right), \quad \forall u=(x, y) \in \widehat{D} .
$$

If the operator equation $u=\widehat{A}_{1} u+\widehat{A}_{2} u$ has a solution $u=(x, y) \in \widehat{D}$, namely,

$$
(x, y)=u=\widehat{A}_{1} u+\widehat{A}_{2} u=\widehat{A}_{1}(x, y)+\widehat{A}_{2}(x, y),
$$

then we obtain

$$
\begin{aligned}
(x, y) & =\widehat{A}_{1} u+\widehat{A}_{2} u \\
& =\left(A_{1} x, A_{1} y\right)+\left(A_{2} y, A_{2} x\right) \\
& =\left(A_{1} x+A_{2} y, A_{1} y+A_{2} x\right) \\
& =(Q(x, y), Q(y, x)) .
\end{aligned}
$$

This implies that the operator $Q(x, y)$ has a coupled fixed point in $\widehat{E}$. We will apply Theorem 3.1 to prove that the operator equation $u=\widehat{A}_{1} u+\widehat{A}_{2} u$ has a solution in $\widehat{E}$. The proof will be given in several steps.

Step I. $\widehat{A}_{1}$ is $\Phi$-orbitally continuous and

$$
\left\|\widehat{A}_{1} w-\widehat{A}_{1} v\right\|_{\widehat{E}}<\|w-v\|_{\widehat{E}}
$$

for all comparable elements $w, v \in \widehat{D}$ with $w \not \equiv v$. 
Since $A_{1}$ is $\Phi$-orbitally continuous, by the definition of $\widehat{A}_{1}$, it is easy to see that $\widehat{A}_{1}$ is $\Phi$-orbitally continuous.

For all comparable elements $w=\left(w_{1}, w_{2}\right), v=\left(v_{1}, v_{2}\right) \in \widehat{D}$ with $w \neq \equiv$, we have

$$
\begin{aligned}
\left\|\widehat{A}_{1} w-\widehat{A}_{1} v\right\|_{\widehat{E}} & =\left\|\left(A_{1} w_{1}, A_{1} w_{2}\right)-\left(A_{1} v_{1}, A_{1} v_{2}\right)\right\|_{\widehat{E}} \\
& =\left\|\left(A_{1} w_{1}-A_{1} v_{1}\right),\left(A_{1} w_{2}-A_{1} v_{2}\right)\right\|_{\widehat{E}} \\
& =\left\|A_{1} w_{1}-A_{1} v_{1}\right\|_{E}+\left\|A_{1} w_{2}-A_{1} v_{2}\right\|_{E} \\
& \leq \varphi\left(\left\|w_{1}-v_{1}\right\|_{E}\right)+\varphi\left(\left\|w_{2}-v_{2}\right\|_{E}\right) \\
& <\left\|w_{1}-v_{1}\right\|_{E}+\left\|w_{2}-v_{2}\right\|_{E} \\
& =\left\|\left(w_{1}-v_{1}, w_{2}-v_{2}\right)\right\|_{\widehat{E}} \\
& =\left\|\left(w_{1}, w_{2}\right)-\left(v_{1}, v_{2}\right)\right\|_{\widehat{E}} \\
& =\|w-v\|_{\widehat{E}} .
\end{aligned}
$$

Hence, we obtain

$$
\left\|\widehat{A}_{1} w-\widehat{A}_{1} v\right\|_{\widehat{E}}<\|w-v\|_{\widehat{E}}
$$

for all comparable elements $w, v \in \widehat{D}$ with $w \neq \equiv v$.

Step II. $\widehat{A}_{2}$ is $\Phi$-orbitally continuous and partially compact.

Since $A_{2}$ is $\Phi$-orbitally continuous, by the definition of $\widehat{A}_{2}$, it is easy to see that $\widehat{A}_{2}$ is $\Phi$-orbitally continuous.

Let $C \subset D$ be a bounded chain. Since $A_{2}$ is partially compact in $D$, it follows that $A_{2}(C)$ is equi-continuous and uniformly bounded in $D$. Set $\widehat{C}=C \times C$. Then $\widehat{C}$ is a bounded chain in $\widehat{D}$. Next, we claim that $\widehat{A}_{2}(\widehat{C})$ is equi-continuous and uniformly bounded in $\widehat{D}$

Since $A_{2}(C) \subset D$ is uniformly bounded, there is a constant $\bar{M}>0$ satisfying $\left\|A_{2} z\right\|_{E} \leq \bar{M}$ for any $z \in C$. For any $Z \in \widehat{A}_{2}(\widehat{C})$, there are $x, y \in C$ satisfying $u=(x, y) \in \widehat{C}$ such that $Z=$ $\widehat{A}_{2} u$. Thus,

$$
\begin{aligned}
\|Z\|_{\widehat{E}} & =\left\|\widehat{A}_{2} u\right\|_{\widehat{E}}=\left\|\left(A_{2} y, A_{2} x\right)\right\|_{\widehat{E}} \\
& =\left\|A_{2} y\right\|_{E}+\left\|A_{2} x\right\|_{E} \\
& \leq 2 \bar{M} .
\end{aligned}
$$

This implies that $\widehat{A}_{2}(\widehat{C})$ is uniformly bounded in $\widehat{D}$.

Since $A_{2}(C)$ is equi-continuous in $D$, for any $z \in C$ and $t_{2}>t_{1}$, we have

$$
\left\|\left(A_{2} z\right)\left(t_{2}\right)-\left(A_{2} z\right)\left(t_{1}\right)\right\|_{E} \rightarrow 0
$$

as $t_{2}-t_{1} \rightarrow 0$. Hence for any $Z=\widehat{A}_{2} u=\widehat{A}_{2}(x, y) \in \widehat{A}_{2}(\widehat{C})$, we have

$$
\begin{aligned}
\left\|Z\left(t_{2}\right)-Z\left(t_{1}\right)\right\|_{\widehat{E}} & =\left\|\left(\widehat{A}_{2} u\right)\left(t_{2}\right)-\left(\widehat{A}_{2} u\right)\left(t_{1}\right)\right\|_{\widehat{E}} \\
& =\left\|\left(\left(A_{2} y\right)\left(t_{2}\right),\left(A_{2} x\right)\left(t_{2}\right)\right)-\left(\left(A_{2} y\right)\left(t_{1}\right),\left(A_{2} x\right)\left(t_{1}\right)\right)\right\|_{\widehat{E}} \\
& =\left\|\left(A_{2} y\right)\left(t_{2}\right)-\left(A_{2} y\right)\left(t_{1}\right),\left(A_{2} x\right)\left(t_{2}\right)-\left(A_{2} x\right)\left(t_{1}\right)\right\|_{\widehat{E}}
\end{aligned}
$$




$$
\begin{aligned}
& =\left\|\left(A_{2} y\right)\left(t_{2}\right)-\left(A_{2} y\right)\left(t_{1}\right)\right\|_{E}+\left\|\left(A_{2} x\right)\left(t_{2}\right)-\left(A_{2} x\right)\left(t_{1}\right)\right\|_{E} \\
& \rightarrow 0
\end{aligned}
$$

as $t_{2}-t_{1} \rightarrow 0$. This implies that $\widehat{A}_{2}(\widehat{C})$ is equi-continuous in $\widehat{D}$.

Therefore, by the Arzela-Ascoli theorem, $\widehat{A}_{2}(\widehat{C}) \subset \widehat{D}$ is relatively compact. Consequently, $\widehat{A}_{2}: \widehat{D} \rightarrow \widehat{D}$ is partially compact.

Step III. There is an element $u_{0} \in \widehat{D}$ satisfying

$$
u_{0} \leq \widehat{A}_{1} u_{0}+\widehat{A}_{2} u
$$

for all $u \in \widehat{D}$.

Let $u_{0}=\left(v_{0}, v_{0}\right)$. For any $u=(x, y) \in \widehat{D}$, by the condition (iii), we have

$$
\begin{aligned}
\widehat{A}_{1} u_{0}+\widehat{A}_{2} u & =\left(A_{1} v_{0}, A_{1} v_{0}\right)+\left(A_{2} y, A_{2} x\right) \\
& =\left(A_{1} v_{0}+A_{2} y, A_{1} v_{0}+A_{2} x\right) \\
& \geq\left(v_{0}, v_{0}\right) \\
& =u_{0} .
\end{aligned}
$$

Hence we obtain the desired conclusion.

Step IV. Every pair of elements in $\widehat{D}$ has an upper and a lower bound.

For every pair of elements $w=\left(w_{1}, w_{2}\right), v=\left(v_{1}, v_{2}\right) \in \widehat{D}$, by condition (iv), there exist $z_{1}, z_{2}, z_{3}, z_{4} \in D$ such that

$$
\begin{aligned}
& z_{1} \leq w_{1}, \quad z_{1} \leq v_{1}, \quad z_{3} \geq w_{1}, \quad z_{3} \geq v_{1}, \\
& z_{2} \leq w_{2}, \quad z_{2} \leq v_{2}, \quad z_{4} \geq w_{2}, \quad z_{4} \geq v_{2} .
\end{aligned}
$$

Thus, we have

$$
\begin{aligned}
& \left(z_{1}, z_{2}\right) \leq\left(w_{1}, w_{2}\right) \leq\left(z_{3}, z_{4}\right), \\
& \left(z_{1}, z_{2}\right) \leq\left(v_{1}, v_{2}\right) \leq\left(z_{3}, z_{4}\right) .
\end{aligned}
$$

Consequently, every pair of elements $w, v \in \widehat{D}$ has an upper and a lower bound.

Therefore, by Theorem 3.1, the operator equation $u=\widehat{A}_{1} u+\widehat{A}_{2} u$ has a solution in $\widehat{E}$.

By Theorems 3.2 and 3.3, the following coupled fixed point theorem is obtained. Because its proof is similar to Theorem 3.3, we omit the details.

Theorem 3.4 Let E be a partially ordered $\Phi$-orbitally complete normed linear space with the norm $\|\cdot\|_{E}$ and the order relation $\leq$, positive cone $K$ be normal, and let $D$ be a nonempty closed subset of $E$. Assume that $A_{1}, A_{2}: D \rightarrow D$ are two monotone nondecreasing mappings satisfying

(i) $A_{1}$ is $\Phi$-orbitally continuous and a partially nonlinear $\mathfrak{D}$-contraction,

(ii) $A_{2}$ is $\Phi$-orbitally continuous and partially compact, 


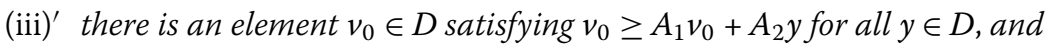

(iv) every pair of elements in D has an upper and a lower bound.

Then $Q(x, y)=A_{1} x+A_{2} y$ has a coupled fixed point in $\widehat{E}$.

Remark 3.5 The hypothesis (iv) of Theorems 3.3 and 3.4 holds if the partially ordered set $E$ is a lattice. We known that the set $C(J, X)$ is a lattice, where $C(J, X)$ is the set of all continuous $X$-valued functions on $J \in \mathbb{R}, X$ is a partially ordered set. For any $x, y \in C(J, X)$, $\max \{x, y\}$ and $\min \{x, y\}$ are the upper and lower bounds, respectively.

Remark 3.6 The assumptions of mixed monotone property and contractive property of the mapping $Q$ are essential in $[2,9,11,16]$. But in Theorems 3.3 and 3.4, we neither assume that the mapping $Q$ is mixed monotone, nor assume that the mapping $Q$ is a contraction. We only suppose that the mapping $Q$ is a nondecreasing mapping and a part of $Q$ (namely, the operator $A_{1}$ ) is a partially nonlinear $\mathcal{D}$-contraction. Plus with other assumptions we obtain the coupled fixed point theorems. Hence Theorems 3.3 and 3.4 extend the main results of $[2,9,11,16]$.

\section{Existence results for fractional evolution systems}

Let $(X,\|\cdot\|)$ be a $\Phi$-orbitally complete normed linear space. Define its positive cone as $\bar{K}=\{x \in X: x \geq 0\}$. Then $X$ becomes a partially ordered $\Phi$-orbitally complete normed linear space with the norm $\|\cdot\|$ and the partial order $\leq$ induced by the cone $\bar{K}$. In this section, we always assume that $\bar{K}$ is normal. Investigate the existence of coupled mild solutions to the initial value problem of the fractional hybrid evolution system

$$
\left\{\begin{array}{l}
{ }^{\mathrm{C}} D_{t}^{\sigma} x(t)+A x(t)=f(t, x(t))+h(t, y(t)), \\
{ }^{C} D_{t}^{\sigma} y(t)+A y(t)=f(t, y(t))+h(t, x(t)), \quad t \in J, \\
x(0)=\tau_{0}, y(0)=\tau_{0} \in X
\end{array}\right.
$$

where $J=[0, b], b>0$ is a constant, ${ }^{\mathrm{C}} D_{t}^{\sigma}$ denotes the $\sigma \in(0,1)$ order Caputo fractional derivative, $-A$ generates a $C_{0}$-semigroup $S(t)(t \geq 0)$ of uniformly bounded linear operator in $X, f$ and $h$ are given functions.

For the $C_{0}$-semigroup $S(t)(t \geq 0)$, if $S(t) x \geq 0$ for all $x \geq 0$, it is called a positive $C_{0}$ semigroup. Throughout this section, we always assume that $-A$ generates a positive $C_{0}$ semigroup $S(t)(t \geq 0)$ of uniformly bounded linear operator in $X$. Namely, there is a constant $M>0$ such that $\|S(t)\| \leq M$ for all $t \geq 0$.

Definitions 4.1 and 4.2 can be found in $[8,10,17]$.

Definition 4.1 The fractional integral of order $\sigma>0$ with the lower limits zero for a function $f \in L^{1}(J, E)$ is defined by

$$
I^{\sigma} f(t)=\frac{1}{\Gamma(\sigma)} \int_{0}^{t} \frac{f(s)}{(t-s)^{1-\sigma}} d s, \quad t>0
$$

where $\Gamma$ is the gamma function. 
Definition 4.2 The Riemann-Liouville derivative of order $n-1<\sigma<n, n \in \mathbb{N}$ with the lower limits zero for a function $f \in L^{1}(J, E)$ can be defined as

$$
{ }^{\mathrm{L}} D_{t}^{\sigma} f(t)=\frac{1}{\Gamma(n-\sigma)} \frac{d^{n}}{d t^{n}} \int_{0}^{t} \frac{f(s)}{(t-s)^{\sigma+1-n}} d s, \quad t>0 .
$$

The Caputo fractional derivative of order $0<\sigma<1$ with the lower limits zero for a function $f \in L^{1}(J, E)$ can be defined as

$$
{ }^{\mathrm{C}} D_{t}^{\sigma} f(t)={ }^{L} D_{t}^{\sigma}(f(t)-f(0)), \quad t>0 .
$$

Define two operator families $\left\{\mathcal{U}_{\sigma}(t)\right\}_{t \geq 0}$ and $\left\{\mathcal{V}_{\sigma}(t)\right\}_{t \geq 0}$ as

$$
\begin{aligned}
& \mathcal{U}_{\sigma}(t) x=\int_{0}^{\infty} \zeta_{\sigma}(\tau) S\left(t^{\sigma} \tau\right) x d \tau \\
& \mathcal{V}_{\sigma}(t) x=\sigma \int_{0}^{\infty} \tau \zeta_{\sigma}(\tau) S\left(t^{\sigma} \tau\right) x d \tau, \quad 0<\sigma<1
\end{aligned}
$$

where

$$
\begin{aligned}
& \zeta_{\sigma}(\tau)=\frac{1}{\sigma} \tau^{-1-\frac{1}{\sigma}} \varrho_{\sigma}\left(\tau^{-\frac{1}{\sigma}}\right), \\
& \varrho_{\sigma}(\tau)=\frac{1}{\pi} \sum_{n=1}^{\infty}(-1)^{n-1} \tau^{-\sigma n-1} \frac{\Gamma(n \sigma+1)}{n !} \sin (n \pi \sigma), \quad \tau \in(0, \infty) .
\end{aligned}
$$

\section{Lemma 4.3}

(i) For any $x \in X$ and fixed $t \geq 0$, one has

$$
\left\|\mathcal{U}_{\sigma}(t) x\right\| \leq M\|x\|, \quad\left\|\mathcal{V}_{\sigma}(t) x\right\| \leq \frac{M}{\Gamma(\sigma)}\|x\|
$$

(ii) If $S(t)(t \geq 0)$ is an equi-continuous semigroup, $\mathcal{V}_{\sigma}(t)$ is equi-continuous in $X$ for $t>0$.

(iii) If $S(t)(t \geq 0)$ is a positive $C_{0}$-semigroup, $\mathcal{U}_{\sigma}(t)$ and $\mathcal{V}_{\sigma}(t)$ are positive operators for all $t \geq 0$.

Proof (i) and (ii) can be found in reference [10, 17]. (iii) is easily seen from the definitions of $\mathcal{U}_{\sigma}(t)$ and $\mathcal{V}_{\sigma}(t)$. So, we omit the details here.

Let $C(J, X)$ be a set of all continuous $X$-valued functions on the interval $J$ and let

$$
\bar{K}_{C}=\{x \in C(J, X): x(t) \in \bar{K}, t \in J\} .
$$

Then $C(J, X)$ is a partially ordered $\Phi$-orbitally complete normed linear space with the norm $\|x\|_{C}:=\sup \{\|x(t)\|: t \geq 0\}$ and the partial order $\leq$ induced by $\bar{K}_{C}$. It is clear that $\bar{K}_{C}$ is normal because $\bar{K}$ is normal.

Let $E=C(J, X)$ and $K=\bar{K}_{C}$. Define two operators $A_{1}, A_{2}: E \rightarrow E$ by

$$
\left(A_{1} x\right)(t)=\mathcal{U}_{\sigma}(t) \tau_{0}+\int_{0}^{t}(t-s)^{\sigma-1} \mathcal{V}_{\sigma}(t-s) f(s, x(s)) d s, \quad t \in J
$$




$$
\left(A_{2} x\right)(t)=\int_{0}^{t}(t-s)^{\sigma-1} \mathcal{V}_{\sigma}(t-s) h(s, x(s)) d s, \quad t \in J
$$

Definition 4.4 An element $(x, y) \in E \times E$ is called a coupled mild solution of the system (4.1) if and only if it satisfies the following system of operator equations:

$$
\begin{cases}x(t)=\left(A_{1} x\right)(t)+\left(A_{2} y\right)(t), & t \in J \\ y(t)=\left(A_{1} y\right)(t)+\left(A_{2} x\right)(t), & t \in J\end{cases}
$$

We shall use Theorem 3.3 to prove that (4.4) has a coupled fixed point in $E \times E$. For this purpose, we consider the following hypotheses:

(H1) The positive $C_{0}$-semigroup $S(t)(t \geq 0)$ is equi-continuous.

(H2) The function $f: J \times X \rightarrow X$ is continuous in $x$ for all $t \in J$ and there exist a constant $\rho \in \mathbb{R}$ with $0<\rho<\frac{\Gamma(\sigma+1)}{M b^{\sigma}}$ and a $\mathfrak{D}$-function $\phi: \mathbb{R}^{+} \rightarrow \mathbb{R}^{+}$with $\phi(r)<r$ for any $r>0$ satisfying

$$
0 \leq f(t, u)-f(t, v) \leq \rho \phi(\|u-v\|), \quad \forall u, v \in X, u \geq v
$$

for all $t \in J$.

(H3) The function $h(t, x): J \times X \rightarrow X$ is continuous, nondecreasing and bounded in $x$.

(H4) There is an element $\bar{x} \in E$ satisfying

$$
\left\{\begin{array}{l}
{ }^{C} D_{t}^{\sigma} \bar{x}(t)+A \bar{x}(t) \leq f(t, \bar{x}(t))+h(t, y(t)), \quad t \in J \\
\bar{x}(0) \leq \tau_{0} \in X
\end{array}\right.
$$

for all $y \in E$.

Since, by (H3), $h(t, x)$ is bounded in $x$ for all $t \in J$, there is a constant $\tilde{M}>0$ such that $\|h(t, x)\| \leq \tilde{M}$ for all $t \in J$ and $x \in X$. For

$$
r^{*} \geq \max \left\{\frac{M \tilde{M} b^{\sigma}}{\Gamma(\sigma+1)},\left(1-\frac{M \rho b^{\sigma}}{\Gamma(\sigma+1)}\right)^{-1}\left(M\left\|\tau_{0}\right\|+\frac{M F^{*} b^{\sigma}}{\Gamma(\sigma+1)}\right)\right\}+1,
$$

where $F^{*}=\sup _{t \in J}\|f(t, 0)\|$, we define an open ball $\mathfrak{B}\left(x_{0}, r\right)$ in $E$ by

$$
\mathfrak{B}\left(x_{0}, r\right)=\left\{x \in E:\left\|x_{0}-x\right\|_{C}<r\right\}
$$

where $r=\left\|x_{0}\right\|_{C}+r^{*}$. Let $D=\overline{\mathfrak{B}}\left(x_{0}, r\right)$. Then $D$ is a closed and bounded subset in $E$. By virtue of the assumptions (H1)-(H3), we have the following lemmas.

Lemma 4.5 Assume that the hypothesis (H2) holds. Then the operator $A_{1}: D \rightarrow D$ is $\Phi$ orbitally continuous, nondecreasing and a partially nonlinear $\mathfrak{D}$-contraction in $E$.

Proof By (H2), (4.2) and (4.5), for any $x \in E$ with $\|x\|_{C} \leq r^{*}$, we have

$$
\begin{aligned}
\left\|\left(A_{1} x\right)(t)\right\| & \leq M\left\|\tau_{0}\right\|+\frac{M}{\Gamma(\sigma)} \int_{0}^{t}(t-s)^{\sigma-1}[\|f(s, x(s))-f(s, 0)\|+\|f(s, 0)\|] d s \\
& \leq M\left\|\tau_{0}\right\|+\frac{M}{\Gamma(\sigma)} \int_{0}^{t}(t-s)^{\sigma-1}\left[\rho \phi\left(\|x\|_{C}\right)+F^{*}\right] d s
\end{aligned}
$$




$$
\begin{aligned}
& \leq M\left\|\tau_{0}\right\|+\frac{M \rho b^{\sigma}}{\Gamma(\sigma+1)} r^{*}+\frac{M F^{*} b^{\sigma}}{\Gamma(\sigma+1)} \\
& \leq r^{*}
\end{aligned}
$$

This implies that $\left\|A_{1} x\right\|_{C} \leq r^{*}$ for any $x \in E$ with $\|x\|_{C} \leq r^{*}$. Moreover, we have

$$
\left\|x_{0}-A_{1} x\right\|_{C} \leq\left\|x_{0}\right\|_{C}+\left\|A_{1} x\right\|_{C} \leq\left\|x_{0}\right\|_{C}+r^{*}=r .
$$

This implies that $A_{1}$ maps $D$ into itself.

Since $S(t)(t \geq 0)$ is a positive $C_{0}$-semigroup, by (H2) and Lemma 4.3, it follows that $A_{1}: D \rightarrow D$ is nondecreasing. Take a sequence $\left\{x_{n}\right\} \subset \Phi\left(x ; A_{1}\right)$ for any $x \in D$ with $x_{n} \rightarrow x^{*}$ as $n \rightarrow \infty$. Since $f$ is continuous in $x$ for all $t \in J$, by assumption (H2) and dominated convergence theorem, we have

$$
\begin{aligned}
\lim _{n \rightarrow \infty}\left(A_{1} x_{n}\right)(t) & =\mathcal{U}_{\sigma}(t) \tau_{0}+\int_{0}^{t}(t-s)^{\sigma-1} \mathcal{V}_{\sigma}(t-s) \lim _{n \rightarrow \infty} f\left(s, x_{n}(s)\right) d s \\
& =\mathcal{U}_{\sigma}(t) \tau_{0}+\int_{0}^{t}(t-s)^{\sigma-1} \mathcal{V}_{\sigma}(t-s) f\left(s, x^{*}(s)\right) d s \\
& =\left(A_{1} x^{*}\right)(t), \quad t \in J .
\end{aligned}
$$

This implies that $A_{1}: D \rightarrow D$ is $\Phi$-orbitally continuous.

For any comparable elements $x, y \in D$, without loss of generality, we assume that $x \geq y$. By (H2), for any $t \in J$, we have

$$
\begin{aligned}
\left\|\left(A_{1} x\right)(t)-\left(A_{1} y\right)(t)\right\| & \leq \int_{0}^{t}(t-s)^{\sigma-1}\left\|\mathcal{V}_{\sigma}(t-s)[f(s, x(s))-f(s, y(s))]\right\| d s \\
& \leq \frac{M \rho}{\Gamma(\sigma)} \int_{0}^{t}(t-s)^{\sigma-1} \phi(\|x(s)-y(s)\|) d s \\
& \leq \frac{M b^{\sigma} \rho}{\Gamma(\sigma+1)} \phi\left(\|x-y\|_{C}\right) .
\end{aligned}
$$

This implies that

$$
\left\|A_{1} x-A_{1} y\right\|_{C} \leq \phi\left(\|x-y\|_{C}\right)
$$

because of $0<\rho<\frac{\Gamma(\sigma+1)}{M b^{\sigma}}$. Hence $A_{1}: D \rightarrow D$ is a partially nonlinear $\mathfrak{D}$-contraction in $E$.

Lemma 4.6 Let the hypotheses (H1) and (H3) hold. Then the operator $A_{2}: D \rightarrow D$ is $\Phi$ orbitally continuous, nondecreasing and partially compact in $E$.

Proof By the assumption (H3), (4.3) and (4.5), for any $x \in E$ with $\|x\|_{C} \leq r^{*}$, we have

$$
\left\|\left(A_{2} x\right)(t)\right\| \leq \frac{M}{\Gamma(\sigma)} \int_{0}^{t}(t-s)^{\sigma-1}\|h(s, x(s))\| d s \leq \frac{M \tilde{M} b^{\sigma}}{\Gamma(\sigma+1)} \leq r^{*} .
$$

This follows that $\left\|A_{2} x\right\|_{C} \leq r^{*}$ for any $x \in E$ with $\|x\|_{C} \leq r^{*}$. Further, we have

$$
\left\|x_{0}-A_{2} x\right\|_{C} \leq\left\|x_{0}\right\|_{C}+\left\|A_{2} x\right\|_{C} \leq\left\|x_{0}\right\|_{C}+r^{*}=r .
$$

This implies that $A_{2}$ maps $D$ into itself. 
Since $S(t)(t \geq 0)$ is a positive $C_{0}$-semigroup, by (H3) and Lemma 4.3, a similar proof as in Lemma 4.5 shows that $A_{2}: D \rightarrow D$ is $\Phi$-orbitally continuous and nondecreasing.

For any $t_{1}, t_{2} \in J$ with $t_{1}<t_{2}$, denote

$$
\begin{aligned}
& G_{1}=\left\|\int_{0}^{t_{1}}\left[\left(t_{2}-s\right)^{\sigma-1}-\left(t_{1}-s\right)^{\sigma-1}\right] \mathcal{V}_{\sigma}\left(t_{2}-s\right) h(s, x(s)) d s\right\|, \\
& G_{2}=\left\|\int_{0}^{t_{1}}\left(t_{1}-s\right)^{\sigma-1}\left[\mathcal{V}_{\sigma}\left(t_{2}-s\right)-\mathcal{V}_{\sigma}\left(t_{1}-s\right)\right] h(s, x(s)) d s\right\|, \\
& G_{3}=\left\|\int_{t_{1}}^{t_{2}}\left(t_{2}-s\right)^{\sigma-1} \mathcal{V}_{\sigma}\left(t_{2}-s\right) h(s, x(s)) d s\right\| .
\end{aligned}
$$

Then, by Lemma 4.3, we have

$$
\begin{aligned}
& G_{1} \leq \frac{M \tilde{M}}{\Gamma(\sigma)} \int_{0}^{t_{1}}\left|\left(t_{2}-s\right)^{\sigma-1}-\left(t_{1}-s\right)^{\sigma-1}\right| d s, \\
& G_{3} \leq \frac{M \tilde{M} b^{\sigma}}{\Gamma(\sigma+1)}\left(t_{2}-t_{1}\right)^{\sigma} .
\end{aligned}
$$

This implies that $G_{1} \rightarrow 0$ and $G_{3} \rightarrow 0$ as $t_{2}-t_{1} \rightarrow 0$. If $t_{1} \equiv 0$ and $0<t_{2} \leq b$, it is clear that $G_{2} \equiv 0$. For $t_{1}>0$ and $\delta \in\left(0, t_{1}\right)$ small enough, we have

$$
\begin{aligned}
G_{2} \leq & \left\|\int_{0}^{t_{1}-\delta}\left(t_{1}-s\right)^{\sigma-1}\left[\mathcal{V}_{\sigma}\left(t_{2}-s\right)-\mathcal{V}_{\sigma}\left(t_{1}-s\right)\right] h(s, x(s)) d s\right\| \\
& +\left\|\int_{t_{1}-\delta}^{t_{1}}\left(t_{1}-s\right)^{\sigma-1}\left[\mathcal{V}_{\sigma}\left(t_{2}-s\right)-\mathcal{V}_{\sigma}\left(t_{1}-s\right)\right] h(s, x(s)) d s\right\| \\
\leq & \frac{\tilde{M}\left(t_{1}^{\sigma}-\delta^{\sigma}\right)}{\sigma} \sup _{s \in\left[0, t_{1}-\delta\right]}\left\|\mathcal{V}_{\sigma}\left(t_{2}-s\right)-\mathcal{V}_{\sigma}\left(t_{1}-s\right)\right\| \\
& +\frac{2 M \tilde{M} \delta^{\sigma}}{\Gamma(\sigma+1)} .
\end{aligned}
$$

Hence $G_{2} \rightarrow 0$ as $t_{2}-t_{1} \rightarrow 0$ and $\delta \rightarrow 0$ because of (H1).

Let $C \subset D$ be an arbitrary chain. For any $Z \in A_{2}(C)$, there is $x \in C$ such that $Z(t)=$ $\left(A_{2} x\right)(t)$ for all $t \in J$. So, for any $t_{1}, t_{2} \in J$ with $t_{1}<t_{2}$, by the definition of the operator $A_{2}$, the inequality

$$
\left\|Z\left(t_{2}\right)-Z\left(t_{1}\right)\right\|=\left\|\left(A_{2} x\right)\left(t_{2}\right)-\left(A_{2} x\right)\left(t_{1}\right)\right\| \leq G_{1}+G_{2}+G_{3}
$$

implies that

$$
\left\|Z\left(t_{2}\right)-Z\left(t_{1}\right)\right\| \rightarrow 0
$$

as $t_{2}-t_{1} \rightarrow 0$. This further implies that $A_{2}(C)$ is equi-continuous on $J$.

On the other hand, by (H3), we have

$$
\|Z(t)\|=\left\|\left(A_{2} x\right)(t)\right\| \leq \int_{0}^{t}(t-s)^{\sigma-1}\left\|\mathcal{V}_{\sigma}(t-s) h(s, x(s))\right\| d s \leq \frac{M \tilde{M} b^{\sigma}}{\Gamma(\sigma+1)}, \quad \forall t \in J
$$


It follows that $\|Z\|_{C} \leq \frac{M \widetilde{M} b^{\sigma}}{\Gamma(\sigma+1)}$. Hence $A_{2}(C)$ is uniformly bounded in $E$. By the Arzela-Ascoli theorem, $A_{2}: D \rightarrow D$ is partially compact.

Theorem 4.7 Let the hypotheses (H1)-(H4) hold. Then the fractional evolution system (4.1) has a coupled mild solution on $J$.

Proof Define two operators $A_{1}$ and $A_{2}$ as in (4.2) and (4.3). By Lemmas 4.5 and 4.6, we deduce that $A_{1}: D \rightarrow D$ is $\Phi$-orbitally continuous, nondecreasing and a partially nonlinear $\mathfrak{D}$-contraction as well as $A_{2}: D \rightarrow D$ is $\Phi$-orbitally continuous, nondecreasing and partially compact.

To apply Theorem 3.3, it remains to prove that there is an element $\bar{x} \in D$ satisfying $\bar{x} \leq$ $A_{1} \bar{x}+A_{2} y$ for all $y \in D$. By (H4), there is an elements $\bar{x} \in D$ satisfying

$$
\left\{\begin{array}{l}
{ }^{\mathrm{C}} D_{t}^{\sigma} \bar{x}(t)+A \bar{x}(t) \leq f(t, \bar{x}(t))+h(t, y(t)), \quad t \in J, \\
\bar{x}(0) \leq \tau_{0} \in X
\end{array}\right.
$$

for all $y \in D$. Let $F(t)=D^{\sigma} \bar{x}(t)+A \bar{x}(t), t \in J$. Then we have

$$
\begin{aligned}
\bar{x}(t) & =\mathcal{U}_{\sigma}(t) \bar{x}(0)+\int_{0}^{t}(t-s)^{\sigma-1} \mathcal{V}_{\sigma}(t-s) F(s) d s \\
& \leq \mathcal{U}_{\sigma}(t) \tau_{0}+\int_{0}^{t}(t-s)^{\sigma-1} \mathcal{V}_{\sigma}(t-s)[f(t, \bar{x}(t))+h(t, y(t))] d s \\
& =\left(A_{1} \bar{x}\right)(t)+\left(A_{2} y\right)(t)
\end{aligned}
$$

for all $y \in D$ and $t \in J$. Hence all the conditions of Theorem 3.3 are satisfied. By Theorem 3.3, the system (4.4) has a coupled fixed point in $\widehat{E}$. Therefore, the fractional evolution system (4.1) has a coupled mild solution in $\widehat{E}$.

By Theorem 4.7, we can obtain the following corollaries easily.

Corollary 4.8 Let the hypotheses (H1), (H3) and (H4) hold. In addition, the following condition is satisfied:

$(\mathrm{H} 2)^{\prime}$ The function $f: J \times X \rightarrow X$ is continuous in $x$ for all $t \in J$ and there is a constant $\beta \in\left(0, \frac{\Gamma(\sigma+1)}{M b^{\sigma}}\right)$ such that

$$
0 \leq f(t, u)-f(t, v) \leq \beta(u-v), \quad \forall u \geq v, t \in J
$$

Then the fractional evolution system (4.1) has a coupled mild solution on $J$.

Corollary 4.9 Let the hypotheses (H1), (H3) and (H4) hold. In addition, the following condition is satisfied:

$(\mathrm{H} 2)^{\prime \prime}$ The function $f: J \times X \rightarrow X$ is continuous in $x$ for all $t \in J$ and there is a constant $\gamma \in\left(0, \frac{\Gamma(\sigma+1)}{M b^{\sigma}}\right)$ such that

$$
0 \leq f(t, u)-f(t, v) \leq \frac{\gamma\|u-v\|}{1+\|u-v\|}, \quad \forall u \geq v, t \in J .
$$

Then the fractional evolution system (4.1) has a coupled mild solution on J. 


\section{Applications}

In this section, we apply the obtained abstract results to the following fractional hybrid dynamic system:

$$
\begin{cases}{ }^{C} D_{t}^{\frac{1}{2}} x(z, t)+\frac{\partial x(z, t)}{\partial z}=f(z, t, x(z, t))+h(z, t, y(z, t)), & (z, t) \in I \times I, \\ { }^{\mathrm{C}} D_{t}^{\frac{1}{2}} y(z, t)+\frac{\partial y(z, t)}{\partial z}=f(z, t, y(z, t))+h(z, t, x(z, t)), \quad(z, t) \in I \times I, & \\ x(0, t)=x(1, t)=0, \quad y(0, t)=y(1, t)=0, \quad t \in I, & \\ x(z, 0)=y(z, 0)=\tau_{0}(z), \quad z \in(0,1), & \end{cases}
$$

where $I=[0,1], h: I \times I \times \mathbb{R} \rightarrow \mathbb{R}$ is defined by

$$
h(z, t, u)= \begin{cases}2, & u \leq 0 \\ 2+\frac{5 u}{1+2 u}, & 0<u<2 \\ 4, & u \geq 2\end{cases}
$$

It is clear that $h: I \times I \times \mathbb{R} \rightarrow \mathbb{R}$ is continuous, nondecreasing and

$$
|h(z, t, u)| \leq 4
$$

This implies that the condition (H3) holds.

Theorem 5.1 Suppose that the following conditions are satisfied:

(P1) The function $f: I \times I \times \mathbb{R} \rightarrow \mathbb{R}$ is continuous and there exist a constant $\rho \in\left(0, \frac{\sqrt{\pi}}{2}\right)$ and $a \mathcal{D}$-function $\phi: \mathbb{R}^{+} \rightarrow \mathbb{R}^{+}$with $\phi(r)<r$ for $r>0$ such that

$$
0 \leq f(z, t, u(z, t))-f(z, t, v(z, t)) \leq \rho \phi(|u(z, t)-v(z, t)|)
$$

for all $(z, t) \in I \times I$ and $u, v \in C(I \times I, \mathbb{R})$ with $u \geq v$.

(P2) There exists a function $\hat{x} \in C(I \times I, \mathbb{R})$ such that

$$
\left\{\begin{array}{l}
{ }^{\mathrm{C}} D_{t}^{\frac{1}{2}} \hat{x}(z, t)+\frac{\partial \hat{x}(z, t)}{\partial z} \leq f(z, t, \hat{x}(z, t))+h(z, t, y(z, t)), \quad(z, t) \in I \times I \\
\hat{x}(z, 0) \leq \tau_{0}(z), \quad z \in(0,1)
\end{array}\right.
$$

for any $y \in C(I \times I, \mathbb{R})$.

Then the fractional hybrid dynamic system (5.1) has a coupled mild solution.

Proof Let $X=C(I, \mathbb{R})$. Then $X$ is a $\Phi$-orbitally complete normed linear space with the norm $\|x(t)\|_{C}=\max _{t \in I}|x(t)|$. Define a positive cone in $X$ by $\bar{K}=\{x \in X: x \geq 0\}$. Then $\bar{K}$ is a closed convex cone in $X$, which is normal. Define an operator $A: D(A) \subset X \rightarrow X$ by

$$
A u=u^{\prime}, \quad u \in D(A):=\left\{u \in X: u^{\prime} \in X, u(0)=u(1)=0\right\} .
$$

It is well known that $A$ generates a $C_{0}$-semigroup $S(t)(t \geq 0)$ given by

$$
S(t) u(z)=u(t+z), \quad t \geq 0, u \in X .
$$


Then $S(t)(t \geq 0)$ is an equi-continuous $C_{0}$-semigroup, but it is not compact, and $\sup _{t \in I}\|S(t)\| \leq 1$. This implies that the condition (H1) holds.

Let

$$
\begin{aligned}
& x(t)(z)=x(z, t), \\
& y(t)(z)=y(z, t), \\
& f(t, x(t))(z)=f(z, t, x(z, t)), \\
& h(t, x(t))(z)=h(z, t, x(z, t)) .
\end{aligned}
$$

Then the fractional hybrid dynamic system (5.1) can be rewritten into the abstract fractional evolution system (4.1).

By the assumptions (P1) and (P2), the conditions (H2) and (H4) hold. Hence by Theorem 4.7, the abstract fractional evolution system (4.1) has a coupled mild solution, which is also the coupled mild solution of the fractional hybrid dynamic system (5.1).

Similarly, using Corollaries 4.8 and 4.9 , we can obtain the following theorems.

Theorem 5.2 Assume that the condition (P2) and the following condition are satisfied:

(P3) The function $f: I \times I \times \mathbb{R} \rightarrow \mathbb{R}$ is continuous and there exist a constant $\beta \in\left(0, \frac{\sqrt{\pi}}{2}\right)$ such that

$$
0 \leq f(z, t, u(z, t))-f(z, t, v(z, t)) \leq \beta(u(z, t)-v(z, t))
$$

for all $(z, t) \in I \times I$ and $u, v \in C(I \times I, \mathbb{R})$ with $u \geq v$.

Then the fractional hybrid dynamic system (5.1) has a coupled mild solution.

Theorem 5.3 Let the condition (P2) and the following condition hold:

(P4) The function $f: I \times I \times \mathbb{R} \rightarrow \mathbb{R}$ is continuous and there exist a constant $\gamma \in\left(0, \frac{\sqrt{\pi}}{2}\right)$ such that

$$
0 \leq f(z, t, u(z, t))-f(z, t, v(z, t)) \leq \frac{\gamma\|u-v\|_{C}}{1+\|u-v\|_{C}}
$$

for all $(z, t) \in I \times I$ and $u, v \in C(I \times I, \mathbb{R})$ with $u \geq v$.

Then the fractional hybrid dynamic system (5.1) has a coupled mild solution.

\section{Acknowledgements}

The first author is thankful to the NSF (No. 11661071) and the third author is thankful to the United States-India Education Foundation, New Delhi, India and IIE/CIES, Washington, DC, USA for Fulbright-Nehru PDF Award (No. 2052/FNPDR/2015).

Competing interests

None of the authors have any competing interests in the manuscript.

Authors' contributions

All authors contributed equally in writing this paper. All authors read and approved the final manuscript.

\section{Author details}

${ }^{1}$ College of Mathematics and Statistics, Northwest Normal University, Lanzhou, 730070, P.R. China. ${ }^{2}$ Department of Mathematics, Texas A\&M University-Kingsville, Kingsville, TX 78363, USA. 


\section{Publisher's Note}

Springer Nature remains neutral with regard to jurisdictional claims in published maps and institutional affiliations.

Received: 24 January 2017 Accepted: 13 July 2017 Published online: 15 August 2017

\section{References}

1. Agarwal, R, El-Gebeily, M, O'Regan, D: Generalized contractions in partially ordered metric spaces. Appl. Anal. 87, 109-116 (2008)

2. Bhaskar, T, Lakshmikantham, V: Fixed point theorems in partially ordered metric spaces and applications. Nonlinear Anal. TMA 65, 1379-1393 (2006)

3. Dhage, B: A nonlinear alternative with applications to nonlinear perturbed differential equations. Nonlinear Stud. 13, 343-354 (2006)

4. Dhage, B: Hybrid fixed point theory in partially ordered normed linear spaces and applications to fractional integral equations. J. Differ. Equ. Appl. 2, 155-184 (2013)

5. Dhage, B: Partially continuous mappings in partially ordered normed linear spaces and applications to functional integral equations. Tamkang J. Math. 45, 397-426 (2014)

6. Granas, A, Dugundji, J: Fixed Point Theory. Springer, New York (2003)

7. Harjani, J, Sadarangani, K: Generalized contractions in partially ordered metric spaces and applications to ordinary differential equations. Nonlinear Anal. TMA 72, 1188-1197 (2010)

8. Kilbas, A, Srivastava, H, Trujillo, J: Theory and Applications of Fractional Differential Equations. North Holland Mathematics Studies, vol. 204. Elsevier, Amsterdam (2006)

9. Lakshmikantham, V, Ćirić, L: Coupled fixed point theorems for nonlinear contractions in partially ordered metric spaces. Nonlinear Anal. TMA 70, 4341-4349 (2009)

10. Liang, J, Yang, H: Controllability of fractional integro-differential evolution equations with nonlocal conditions. Appl. Math. Comput. 254, 20-29 (2015)

11. Luong, N, Thuan, N: Coupled fixed points in partially ordered metric spaces and application. Nonlinear Anal. TMA 74, 983-992 (2011)

12. Nieto, J, Rodriguez-López, R: Contractive mapping theorems in partially ordered sets and applications to ordinary differential equations. Order 22, 223-239 (2005)

13. Nieto, J, Rodriguez-López, R: Existence and uniqueness of fixed point in partially ordered sets and applications to ordinary differential equations. Acta Math. Sin. Engl. Ser. 23(12), 2205-2212 (2007)

14. O'Regan, D, Petrusel, A: Fixed point theorems for generalized contractions in ordered metric spaces. J. Math. Anal. Appl. 341, 1241-1252 (2008)

15. Ran, A, Reurings, M: A fixed point theorem in partially ordered sets and some applications to metric equations. Proc. Am. Math. Soc. 132, 1435-1443 (2003)

16. Samet, B: Coupled fixed point theorems for a generalized Meir-Keeler contraction in partially ordered metric spaces. Nonlinear Anal. TMA 72, 4508-4517 (2010)

17. Zhou, Y, Jiao, F: Nonlocal Cauchy problem for fractional evolution equations. Nonlinear Anal., Real World Appl. 11, 4465-4475 (2010)

\section{Submit your manuscript to a SpringerOpen ${ }^{\circ}$ journal and benefit from:}

- Convenient online submission

- Rigorous peer review

- Open access: articles freely available online

- High visibility within the field

- Retaining the copyright to your article

Submit your next manuscript at $\gg$ springeropen.com 